\title{
SUBDIVISION-BASED \\ MESH REFINEMENT \\ FOR RAPID PROTOTYPING
}

\author{
Suzuki, Hiromasa \\ The University of Tokyo \\ Hongo 7-3-1, Bunkyo, Tokyo 113-8656 \\ Japan \\ suzuki@cim.pe.u-tokyo.ac.jp
}

Yamamoto, Tetsuya

Toshiba Corp.

Kanai, Takashi

Keio University

Kimura, Fumihiko

The University of Tokyo

\begin{abstract}
In this paper a method for refining a triangular mesh is proposed in which more vertices and faces are added to the mesh to make it smoother. We apply this method to a problem in the field of rapid prototyping wherein triangular meshes are major shape representations. Based on the concept of subdivision surface, we propose a new refinement method called Local Surface Fitting Scheme (LSF Scheme). There the refinement is made by referring to the quadric surfaces locally defined to fit the neighborhood of vertices in the triangular mesh. In order to evaluate this scheme, it is compared with Zorin's Modified Butterfly scheme (MB Scheme) [20]. A prototype system is developed and some examples are demonstrated. The results show that the LSF Scheme can produce smooth triangular meshes. We apply this scheme to a problem in rapid prototyping.
\end{abstract}

Keywords: Mesh refinement, subdivision surface modeling, triangular mesh, surface interpolation, rapid prototyping 


\section{INTRODUCTION}

In this paper, a method for refining a triangular mesh is proposed wherein more vertices and faces are added to the mesh to make it smoother. We are particularly interested in its application to rapid prototyping, a technology for producing a physical object directly and rapidly from three dimensional shape data, usually in the form of a triangular mesh. The prototyping process is based on so-called layered manufacturing technologies, which generate objects by piling up thin layers.

For rapid prototyping, the geometric model for an object to be produced is generated in the form of surface or solid models using CAD systems. In many cases the model is triangulated into a mesh and stored in a file of de facto standard format of STL. This file is transferred to the rapid prototyping system which then slices the triangular mesh along the vertical axis into a series of horizontal sections of specified thickness. These sections are used for producing thin layers of the physical prototype.

This process involves many interesting geometrical issues which have been studied extensively these years[1]. One of the major concerns is the surface smoothness of the prototyped objects, which is affected by various factors. One is the triangulation process in which the shape of the part originally represented by smooth surfaces is approximated in a triangular mesh. In case a prototyped object does not have a required smoothness, we have to refine the mesh by adding more vertices and faces. For such refinement, finer triangulation must be redone at the CAD system using the original surface data.

However, in some situations, the original surface data is not available for various reasons. For instance, rapid prototyping is often made at a specialized service bureau outside the company and the bureau is given only the triangular mesh data from the company but not the original surface data. The same problem occurs when the size of the object is enlarged. We will describe such example in Section 4.

It is practically useful if we can produce a finer mesh without the surface data. But this seems theoretically impossible because the mesh conveys little information about its original surface. However, by assuming a certain association between the triangular mesh and its underlying surface, we can properly refine the mesh.

One of such associations is that between a parametric surface and its control polygon. We can refine the control polygon by knot insertion or by patch subdivision. Such a relationship has been recently studied in 
the field of subdivision surface modeling. We apply this concept to the above mesh refinement problem.

In Section 2, a brief introduction to subdivision surface modeling is given. In Section 3, based on the concept of subdivision surface, we propose a new subdivision scheme called Local Surface Fitting Scheme (LSF Scheme) wherein the refinement is made by referring to the quadric surface that locally fits the neighborhood of a vertex in the triangular mesh. In order to evaluate this scheme, it is compared with Zorin's Modified Butterfly scheme (MB Scheme)[20] using examples in Section 4.

\section{SUBDIVISION SURFACE MODELING 2.1. LOOP SUBDIVISION SCHEME}

Catmull and Clark[2] and Doo and Sabin[4] first proposed subdivision for surface modeling. The basic idea is to define a smooth surface by repeatedly and infinitely subdividing the polygon according to a rule. For instance in the Catmull-Clark subdivision scheme, a (regular) quadrilateral mesh is known to converge to the bicubic B-spline surface taking that initial mesh as the control mesh. Many subdivision schemes have been invented so far. Here we will use the Loop scheme[11] to introduce some basic features of subdivision surfaces, because it is simple and defined specifically for triangular meshes.

Generally, one subdivision step consists of two substeps: splitting and positioning. At the splitting step, new vertices are added to the graph structure of the polygonal mesh. Then those vertices and often old vertices are located by the positioning step. Those two steps are then repeated.

In the Loop scheme, and also in many other schemes based on the triangular mesh, a four-to-one splitting scheme is used for subdividing the triangular mesh, as shown in Fig. 1. A vertex is inserted into every edge, and the edges are split into two. Then a triangular face is split into four smaller triangles. Thus the number of the mesh faces is increased four times. Those inserted vertices are called odd vertices, while the old vertices are called even vertices.

The number of edges incident to a vertex is called valence. If all the vertices of a triangular mesh have valence of six, the mesh is regular. A vertex whose valence is not six is called extraordinary vertex. All the odd vertices have valence of six, while the extraordinary vertex remains so after subdivision steps.

In the positioning step, the positions of vertices are computed. (In some scheme the even vertices are not changed, as will be discussed 
later.) The vertex position is defined by a linear combination of nearby vertices. In the Loop scheme the position of the odd vertices is computed as follows:

$$
\frac{1}{8}\left(3 P_{1}+3 P_{2}+P_{3}+P_{4}\right)
$$

where $P_{1}$ and $P_{2}$ are the vertices of the edge, and $P_{3}$ and $P_{4}$ are the other two vertices of the triangles sharing this edge.

The position of an even vertex is defined by:

$$
(1-k \beta) P+\beta\left(P_{1}+P_{2}+\cdots+P_{k}\right),
$$

where $P$ is the position of the even vertex before subdivision, and $P_{i}$ are its incident vertices before subdivision. $k$ is the valence of the central vertex.

In both cases, the positions are defined by averaging incident vertices with weights. These splitting pattern and weighted averaging formulas are graphically represented by diagrams called mask or stencil, as shown in Fig. 2.

The Loop scheme is based on a three-directional box spline, and for such spline-based subdivision schemes, the masks are derived completely from the surface functions. Loop proposed to use the coefficients shown in the figure, and these coefficients guarantee that the limit surface of the scheme is $\mathrm{C}^{2}$-continuous except at so-called extraordinary vertices at which it is $\mathrm{C}^{1}$-continuous.

In this scheme, both even and odd vertices are relocated. However, in some other scheme, only the odd vertices are relocated and even vertices are kept at the same positions. This type of subdivision scheme is called interpolating scheme, because the limit surface goes through the vertices of the initial mesh. The former type of schemes including the Loop scheme are called approximating, which does not go through the initial mesh but approximates them.

\subsection{RELATED WORK}

The papers of Doo and Sabin[4] and Catmull and Clark [2] were the first papers describing subdivision algorithms for surfaces. Though not much work was done regarding this problem in the 1980s and in the beginning of 1990 s, recently the ability of subdivision surface for representing free form shapes has been recognized and many new theoretical and practical results have been obtained. New schemes were proposed $[20,9,13]$, and general theory was developed [14]

Hoppe [8] proposed extension of subdivision masks for flexibly modeling a variety of shapes. This year DeRose presented a paper on the extensive use of the Catmull-Clark surface for computer animation[3]. A 

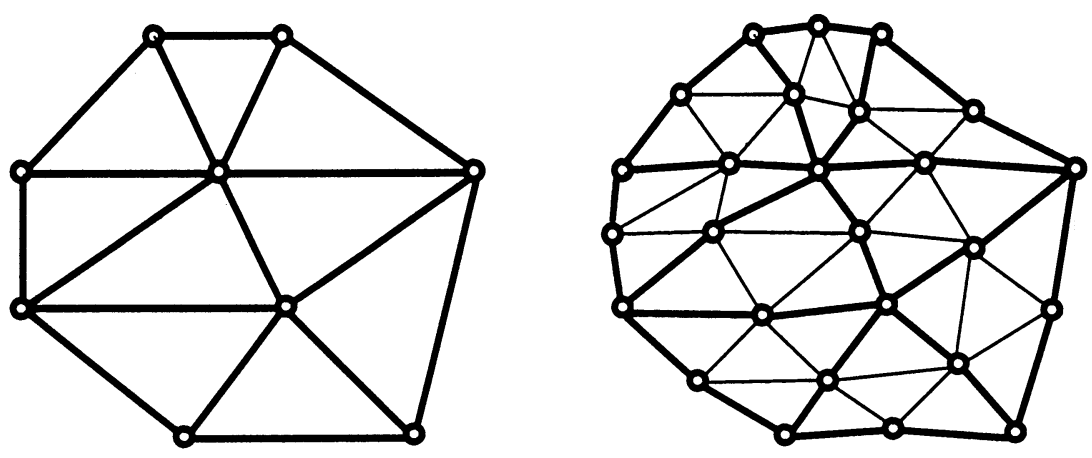

Figure 1 Four-to-one Subdivision.

$3 / 8$

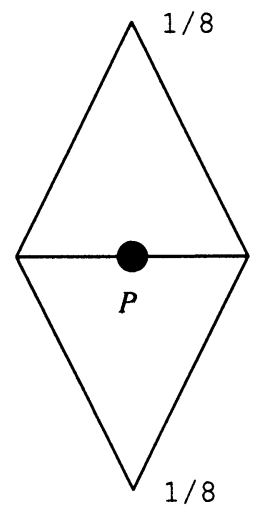

$3 / 8$

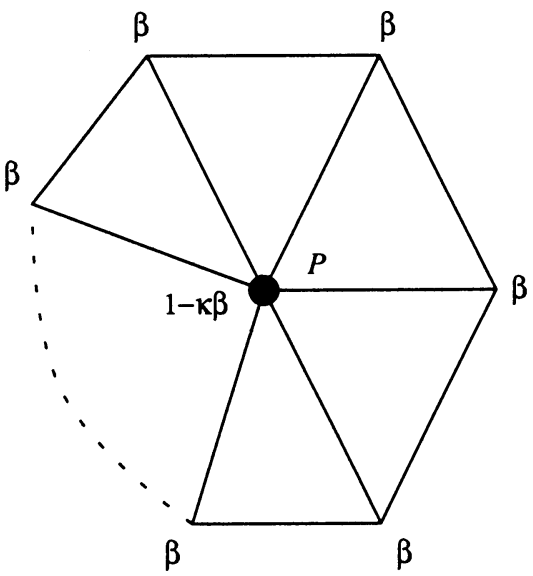

Figure 2 Subdivision Mask. $k$ is the valence of $P$ and $\beta$ is a constant.

comprehensive tutorial course was also organized by Schröder and Zorin [15]. Particularly, its second chapter written by Zorin has provided much of the basis for this paper.

\subsection{INTERPOLATING SCHEME}

Consider the problem described in Section 1. Triangular meshes for rapid prototyping are generated by triangulating CAD models, and their vertices are sampled from the models' surfaces. So we choose interpolating subdivision schemes because they retain the positions of the initial vertices.

A few interpolating schemes have been proposed. Nasri[12] extends the Doo-Sabin scheme so as to be interpolative. Halstead[7] also modifies 
the Cutmull-Clark scheme to satisfy position and normal constraints. However, both need to solve simultaneous linear equations which are global to the surface. As local schemes, Kobbelt[10] presented an interpolating scheme for quadrilateral meshes.

For triangular meshes, the Butterfly scheme $[5,6]$ is local and interpolating. Although the Butterfly scheme can be defined on arbitrary triangular meshes, the limit surface is not $\mathrm{C}^{1}$-continuous at vertices of valence $k=3$ and $k>7$, while it is $\mathrm{C}^{1}$ on regular meshes.

A Modified Butterfly Scheme (MB Scheme) has been proposed by Zorin[20]. This modification makes it possible for the scheme to produce $\mathrm{C}^{1}$-continuous surfaces for arbitrary meshes. The masks for the scheme are shown in Fig. 3.

In these interpolating schemes, meshes converge to the limit surfaces, but they do not have closed form representation, unlike approximating schemes based on splines. But this does not mean that we can not evaluate the surface at a point. We can even compute the normal vectors directly from the mesh.

It is worth noting that the convergence of the subdivision process is very fast. So even with subdividing several times, we can attain a smooth mesh which is near to the limit surface.

In the next section we propose our own interpolating subdivision scheme for irregular mesh setting as is the case of the MB scheme. And we will compare the generated surfaces with those two schemes.

\section{LOCAL SURFACE FITTING SCHEME}

Local Surface Fitting Scheme (LSF Scheme) is our proposed subdivisionbased refinement scheme[16]. It is defined for triangular meshes, and the four-to-one subdivision is used. What is particular to this scheme is that the position of an inserted vertex (odd) is determined by referring to the quadric surfaces, each of which locally fits to a neighborhood around an even vertex. We use a method described by Welch and Witkin[18] to define the surface. Its brief description is given in Appendix.

This quadric surface $\mathbf{s}(u, v)$ defined at each even vertex is parameterized in such a way that $\mathbf{s}(0,0)$ and $\mathbf{s}\left(u_{i}, v_{i}\right)$ correspond to $V$ and the neighborhood point $P_{i}$, respectively. So we can compute a midpoint of the edge curve $V P_{i}$ as $\mathbf{s}\left(u_{i} / 2, v_{i} / 2\right)$ as shown in Fig. 4.

From another quadric surface $\mathbf{s}^{\prime}(u, v)$ centered at $P_{i}$, we compute another midpoint position $\mathbf{s}^{\prime}\left(u_{j} / 2, v_{j} / 2\right)$ for the edge curve $P_{i} V$ in the same way. Then the average of those two midpoints $\mathbf{s}\left(u_{i} / 2, v_{i} / 2\right)$ and $\mathbf{s}^{\prime}\left(u_{j} / 2, v_{j} / 2\right)$ is taken to be the position of the odd vertex inserted into this edge. 

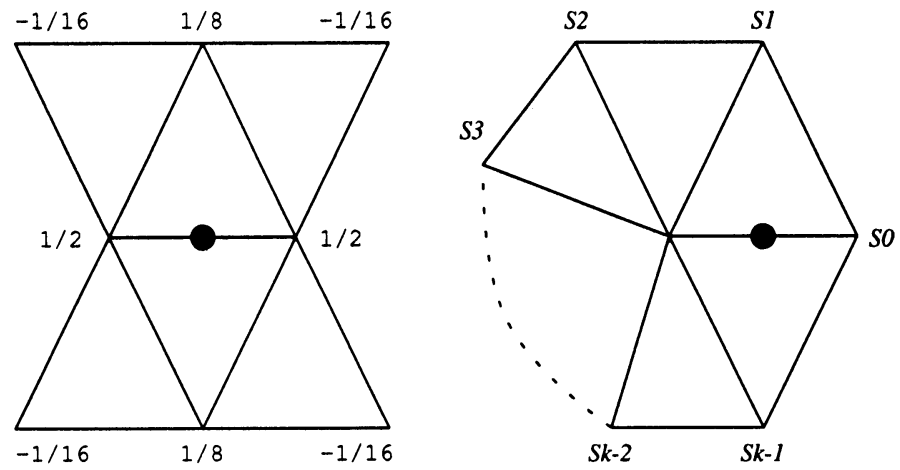

Figure 3 Modified Butterfly Scheme (MB Scheme). Left: mask for odd vertices with regular neighbors. Right: Mask for odd vertices adjacent to an extraordinary vertex. The coefficients $S_{i}$ are $\frac{1}{k}\left(\frac{1}{4}+\cos \frac{2 i \pi}{k}+\frac{1}{2} \cos 4 i \pi k\right)$ for $k \geq 5$. For $k=3, S_{0}=\frac{1}{12}$, $S_{1,2}=-\frac{1}{12} ;$ for $k=4, S_{0}=\frac{3}{8} S_{2}=-\frac{1}{8} ; S_{1,3}=0$.

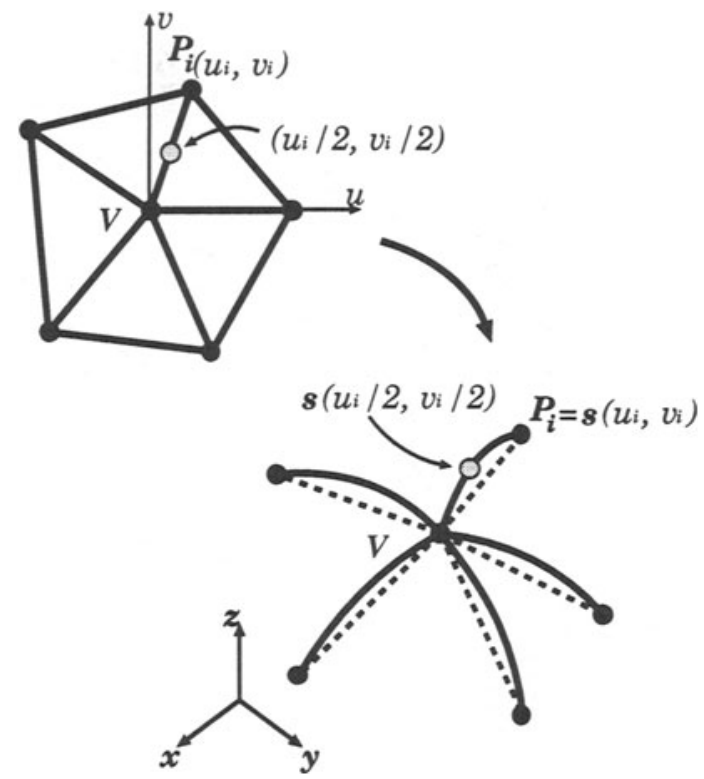

Figure 4 Interpolation using Local Surface Approximation.

In comparison with the $\mathrm{MB}$ scheme, the computation for the odd vertices is more complicated. We do not know if we can regard this subdivision scheme as a subdivision surface, because it is not yet shown that our subdivision scheme converges to a surface or not. However, 
for our purpose we are not interested in the limit surface, and it is sufficient if the scheme generates an intuitively smooth mesh after several subdivision steps at most.

\section{EVALUATION AND EXAMPLE 4.1. SMOOTHNESS EVALUATION}

We apply both the MB scheme and our LSF scheme to a simple example shown in Fig. 5. Note that the triangulation of the cylinder in Fig. 5 is not regular; that is, the valence of some vertices is not six. The results are shown in Figs. 6 and 7. Those are meshes generated by three steps of subdivision. The shaded image in Fig. 6 shows some undulation, which is not obvious in Fig. 7.

In order to analyze this undulation, the meshes are sectioned by a plane and the smoothness of the sectioning curves is examined, as shown in Fig. 8. These curves are piecewise linear, and we parameterize them using chord length. For a piecewise linear curve with nodes $\mathbf{p}_{i}, \quad(i=$ $1, \ldots, N)$, the curve parameter $t_{i}$ of $\mathbf{p}_{i}$ is defined as:

$$
t_{i}=\frac{\sum_{k=1}^{i} e_{k}}{\sum_{k=1}^{N} e_{k}}
$$

where $e_{k}$ is the length of $k$-th segment $\left\langle\mathbf{p}_{k}, \mathbf{p}_{k+1}\right\rangle, \quad\left(\mathbf{p}_{N+1}=\mathbf{p}_{1}\right)$.

With this parameterization, we can define a piecewise linear interpolation function $\mathbf{f}(t)(0 \leq t \leq 1)$ such that $\mathbf{p}_{i}=\mathbf{f}\left(t_{i}\right)\left(0 \leq t_{i} \leq 1\right)$. As each interval of $\left[t_{i}, t_{i+1}\right]$ is small enough, the first order derivative can be expressed approximately as:

$$
\mathbf{f}^{\prime}\left(t_{i}\right) \simeq \frac{\mathbf{f}\left(t_{i-1}\right)-\mathbf{f}\left(t_{i}\right)}{t_{i-1}-t_{i}}=\frac{\mathbf{p}_{i-1}-\mathbf{p}_{i}}{t_{i-1}-t_{i}}
$$

If the derivative $\mathbf{f}^{\prime}(t)$ varies smoothly, this curve $\mathbf{f}(t)$ approximately achieves $C^{1}$ continuity.

By transforming the sectioning curves in Fig. 8 to the $x y$ plane with their centers located at the origin, we obtain planar sectioning curves. The graphs in Fig. 9 show the derivative of the coordinate $x, y$ of those sectioning curves.

In this particular example, we observe some undulations in the MB scheme. We think such undulations are produced when the initial mesh is irregular, or more specifically, when the neighborhood vertices are unevenly distributed around a vertex. In such a case, the position of the odd vertices may be biased in the direction where neighborhood vertices are densely populated, because of the isotropy of the mask of the MB 

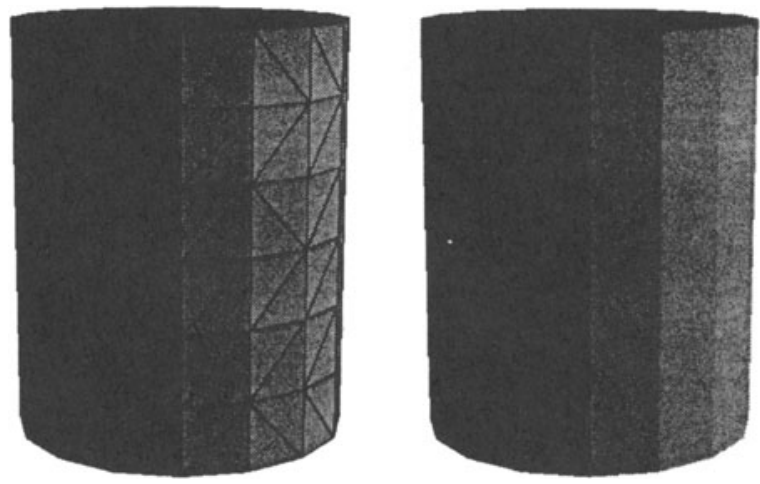

Figure 5 Cylindrical Mesh.
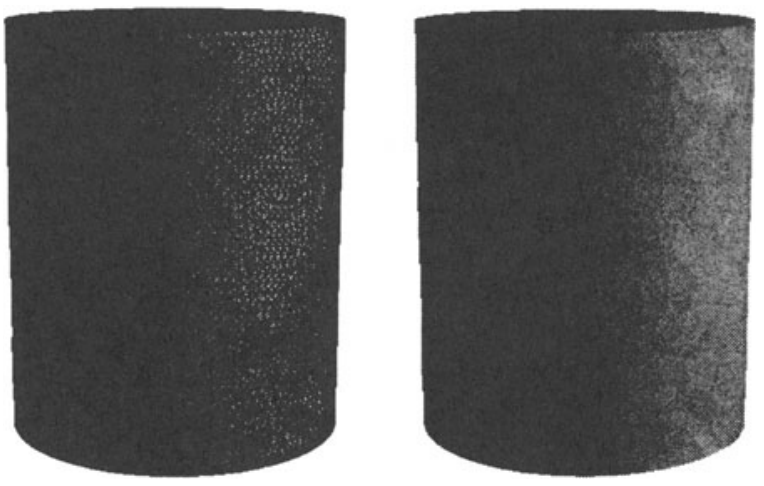

Figure 6 Subdivision by MB Scheme.
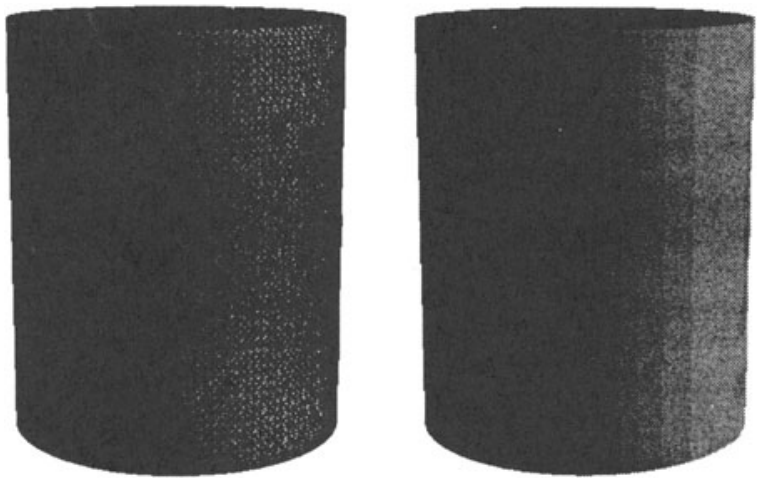

Figure 7 Subdivision by LSF Scheme. 


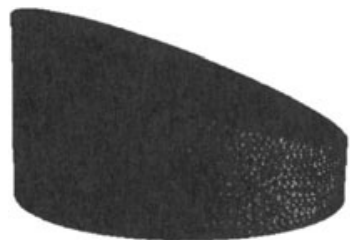

(a) MB Scheme

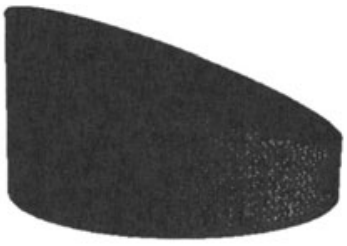

(b) LSF Scheme

Figure 8 Cross Sections of Subdivided Meshes.

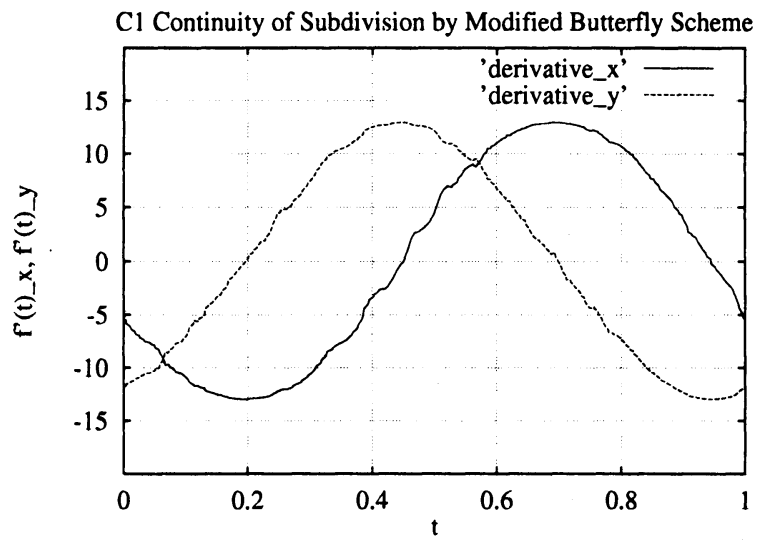

(a) MB Scheme

C1 Continuity of Subdivision by Local Surface Fitting Scheme

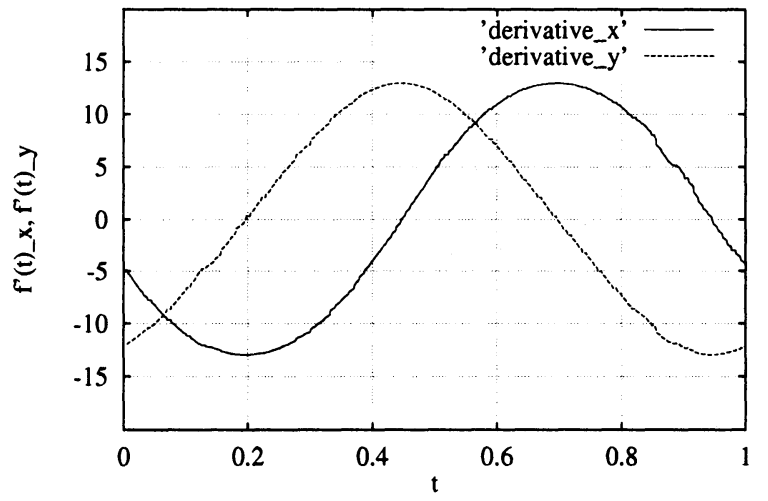

(b) LSF Scheme

Figure 9 Continuity evaluation. 
scheme, as shown in Fig. 3. In the LSF scheme, such effect of the uneven distribution may be absorbed by the parameterization method for the neighborhood of a vertex (see Appendix).

The computation time of the MB scheme (three steps) is about 2.5 seconds on an SGI workstation (Power Indigo2 /MIPS R8000), while the LSF scheme takes about 5.2 seconds.

\subsection{RAPID PROTOTYPING EXAMPLES}

Figure 10 shows iron models. Figure 10 (a) is the initial mesh and (b) is the mesh refined by subdividing twice. The shaded pictures represent Gaussian curvature distribution wherein the darker color shows higher curvature. The curvature at a vertex is calculated from the quadric surface defined by the method described in Appendix.

We extended our approach to deal with such sharp crease edges as exist between the side and bottom surfaces[16, 17]. Those edges are subdivided so that they are rounded only in the direction along the crease, and the sharp angle across the crease is reserved. Furthermore, nonuniform subdivision is realized so that subdivisions are made dependent on the curvature of the local surfaces.

In both (b) and (c), the meshes are smoothly refined in keeping with the characteristics of the initial mesh. Figure 11 shows a prototype model generated using the mesh (b) in Fig. 10. By touching the surfaces of this prototype we could hardly feel the edges in the texture that we felt in the other prototype generated from the initial mesh.

Figure 12 shows an example for a tiger model and Fig. 13 shows its rapid prototypes. The smaller model is created from the original mesh model and the larger model is made from the refined mesh model. If the larger tiger model was made from the original mesh model, the prototype would have a coarse surface. Such scaling up of models is not rare, because mechanical prototypes are often produced in certain scaling factors of $1 / 2,1 / 10$ etc. and can be arbitrarily scaled for such artistic ornaments as the tiger model. The mesh refinement is useful for those cases.

\section{CONCLUSION}

In this paper, the LSF scheme, a subdivision method for refining a triangular mesh, is proposed. The method resembles subdivision surface modeling, though as yet it has no sound theoretical basis. However, by applying it to several examples, and by comparing with the wellknown Modified Butterfly subdivision scheme, we find that the scheme can generate properly smooth meshes. Our future work is aimed at 

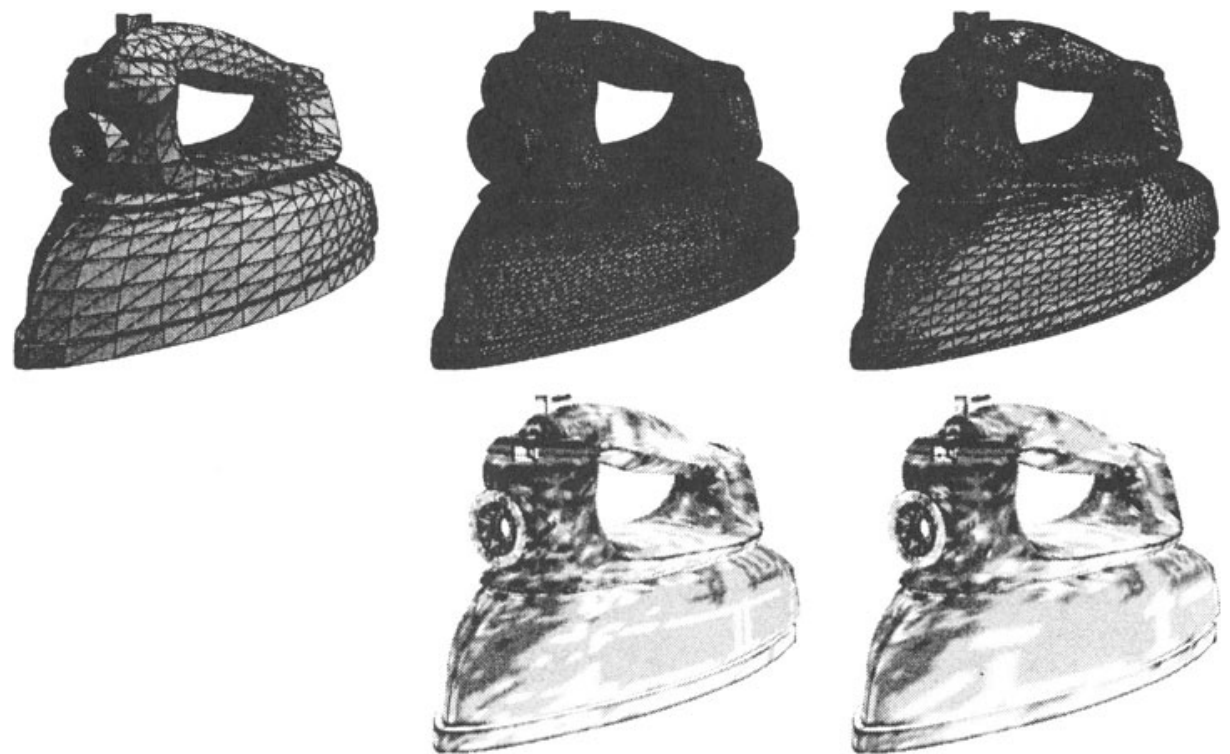

(a) Initial Mesh

(b) LSF Scheme

(c) Non Uniform LSF

Figure 10 Iron Models: (a) Initial(vertex: 1110, face: 2212), (b) Subdivided twice (vertex: 17770, face: 35392), and (c) Non-uniformly subdivided (vertex: 12289, face: 24570).

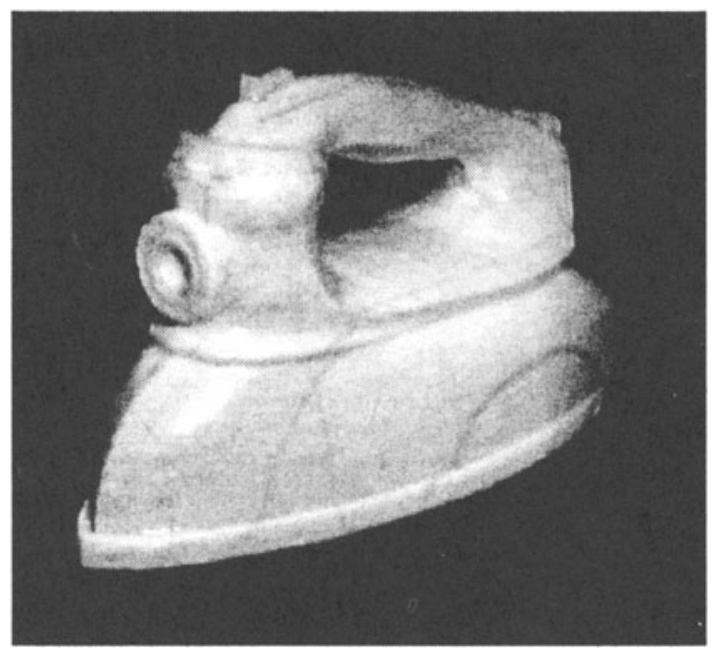

Figure 11 Prototype Model for Iron Model in Figure 10 (b). 

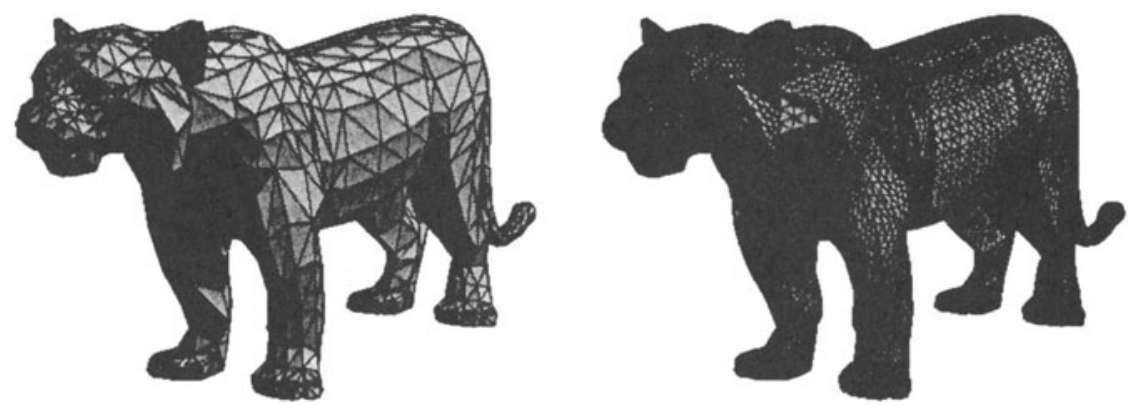

Figure 12 Tiger Models: Left: Initial (vertex: 956, face: 1908), Right: subdivided twice (vertex: 15266, face: 30528).

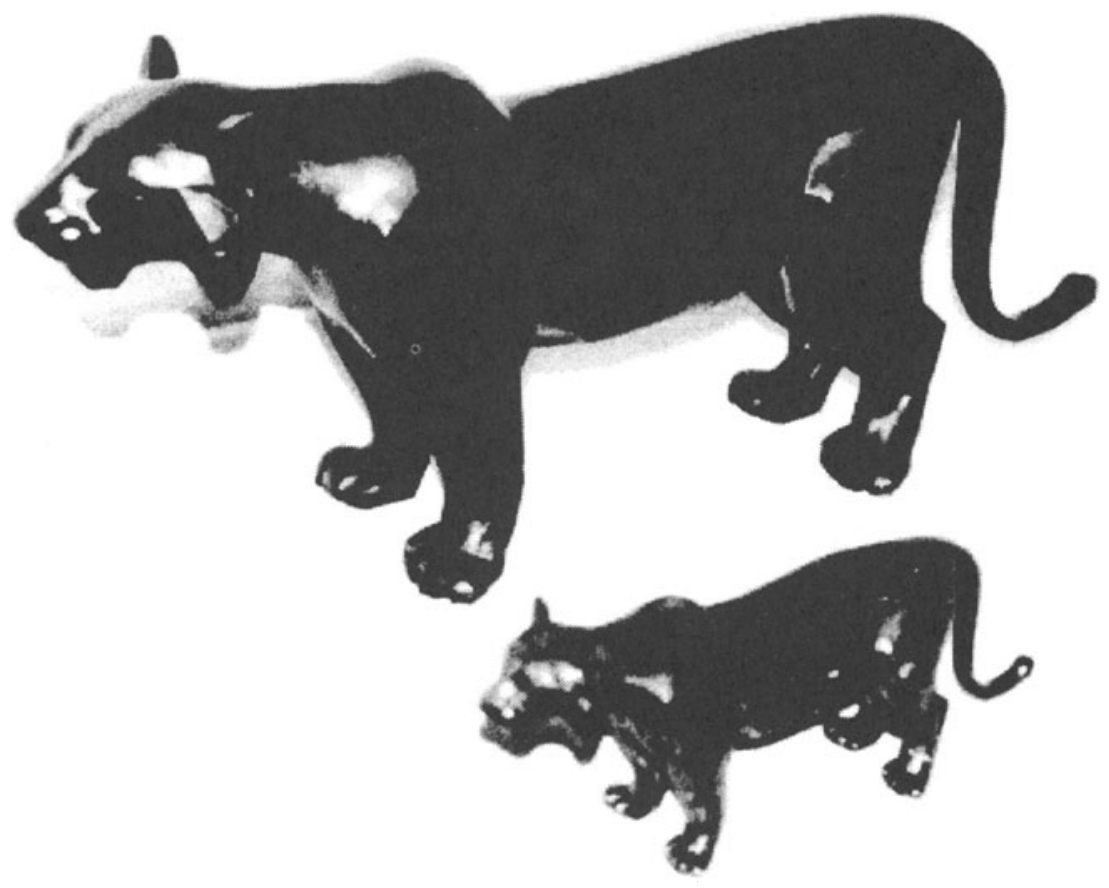

Figure 13 Prototype Models for Tiger Model. 
further analysis of the scheme; specifically, we intend to investigate its limit surface. We also plan to extend the scheme to mesh editing.

\section{Acknowledgement}

We would like to thank Toyoda Machine Works, Ltd. Aichi, Japan and Armonicos Co., Ltd., Shizuoka, Japan. Toyoda Machine Works allowed rapid prototyping of the iron models using their machines. Armonicos created the tiger models using their rapid prototyping software. A part of this research is funded by The Okawa Foundation for Information and Telecommunications, Tokyo, Japan.

\section{Appendix}

We briefly introduce a surface-fitting method developed by Welch and Witkin[18]. In their method, a truncated Taylor series expansion is fitted at a neighborhood around a point. First, a neighborhood parameterization for surface-fitting is constructed. The vertices incident to $V$ are called neighborhood point, and their set $N(V)$ is defined as the neighborhood of $V . V$ is called the center of the neighborhood (Fig. 14). The neighborhood points $P_{i} \in N(V)$ are indexed around the center in the counterclockwise direction. For convenience sake, $P_{0} \equiv V$.

For constructing a neighborhood parameterization, the angular separation between each neighboring point is measured. As shown in Fig. 15, $\theta_{i}$ denotes the angle $\angle P_{i} V P_{i+1}$, where $i+1$ is computed in modulo $n$. A scaling factor $\alpha$ is computed for scaling those angles so that their sum is $2 \pi$ :

$$
\alpha\left(\theta_{1}+\theta_{2}+\theta_{3}+\cdots+\theta_{n}\right)=2 \pi .
$$

We define the polar coordinates of $P_{i}$ as $\left(r_{i}, \phi_{i}\right)$, where $r_{i} \equiv\left|P_{i} V\right|$ and $\phi_{i} \equiv \sum_{j=1}^{i-1} \alpha \theta_{j}$. By converting this polar coordinate into Cartesian coordinates, we obtain the surface parameter for $P_{i}$ as $\left(u_{i}, v_{i}\right)=$ $\left(r_{i} \cos \left(\phi_{i}\right), r_{i} \sin \left(\phi_{i}\right)\right)$.

Then the surface $\mathbf{s}(u, v)$ is generated so that the surface point $\mathbf{s}\left(u_{i}, v_{i}\right)$ corresponds to $P_{i}$. $\mathbf{s}(u, v)$ is given by the following equation:

$$
\begin{aligned}
\mathbf{s}(u, v) & =c_{0}+c_{1} u+c_{2} v+c_{3} \frac{u^{2}}{2}+c_{4} u v+c_{5} \frac{v^{2}}{2} \\
& =\mathbf{b}(u, v) \mathbf{c}^{T}
\end{aligned}
$$

where $\mathbf{b}(u, v)$ is the basis row vector $\left(1, u, v, \frac{1}{2} u^{2}, u v, \frac{1}{2} v^{2}\right)$, $\mathbf{c}=\left[c_{0}, c_{1}, c_{2}, c_{3}, c_{4}, c_{5}\right]$ and $\mathbf{c}$ a column vector of coefficients. 


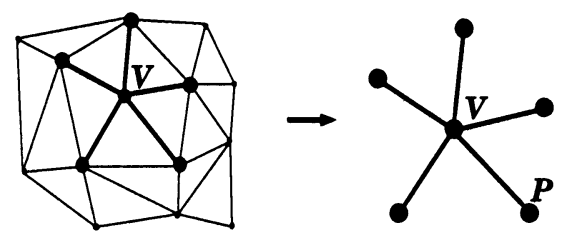

Figure 14 Neighborhood of $V$ ( $V=$ center, $P$ a neighborhood point).

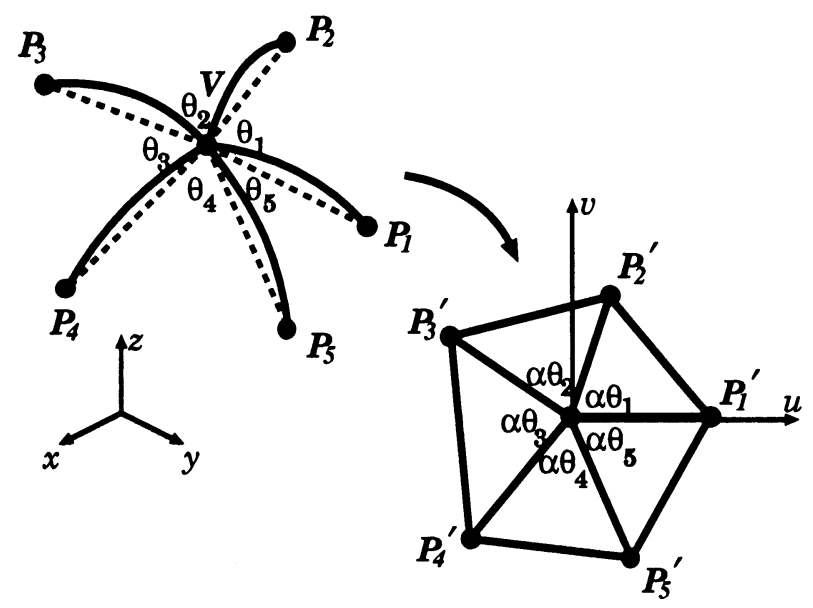

Figure 15 Projection of Neighborhood Points: $\alpha\left(\theta_{1}+\theta_{2}+\theta_{3}+\theta_{4}+\theta_{5}\right)=2 \pi$

From $\mathbf{s}\left(u_{0}, v_{0}\right) \equiv \mathbf{s}(0,0)=P_{0}(=V)$, we can easily show $c_{0}=P_{0}$. The remaining coefficients will be determined such that $\mathbf{s}\left(u_{i}, v_{i}\right)$ are a least-squares fit to the $P_{i}$.

Shifting the origin to $P_{0}$ yields the vector $\mathbf{q}=\left[P_{1}-P_{0}, \ldots, P_{n}-\right.$ $\left.P_{0}\right]^{T}$. The matrix $\mathbf{S}$ for this shifted, center-constrained system is built by evaluating the basis vector $\mathbf{b}\left(u_{i}, v_{i}\right)$ for each of the neighbors and collecting these rows into a matrix, then deleting its first column. Then matrix $\mathbf{S}$ is defined:

$$
\mathbf{S} \equiv\left[\begin{array}{ccccc}
u_{1} & v_{1} & \frac{1}{2} u_{1}^{2} & u_{1} v_{1} & \frac{1}{2} v_{1}^{2} \\
& & \vdots & & \\
u_{n} & v_{n} & \frac{1}{2} u_{n}^{2} & u_{n} v_{n} & \frac{1}{2} v_{n}^{2}
\end{array}\right] .
$$

Using this $\mathbf{S}$ and $\mathbf{q}$, we obtain $\mathbf{S c}^{T}=\mathbf{q}$, and the least-squares solution for $\mathbf{c}$ is:

$$
\mathbf{c}^{T}=\left[\mathbf{S}^{T} \mathbf{S}\right]^{-1} \mathbf{S}^{T} \mathbf{q}=\mathbf{Z} \mathbf{q}
$$


By substituting Equation (8) to Equation (6), $\mathbf{s}(u, v)$ is obtained. We shift the origin back by replacing $Q_{i}$ with $P_{i}$ :

$$
\mathbf{s}(u, v)=\mathbf{b}(u, v)\left[\begin{array}{cc}
1 & \mathbf{o} \\
-\mathbf{z} & \mathbf{Z}
\end{array}\right] \mathbf{P}=\mathbf{b}(u, v) \mathbf{B P}
$$

where $\mathbf{P} \equiv\left[P_{0}, P_{1}, \ldots, P_{n}\right]^{T}$. Each element of $\mathbf{z}$ is the sum of a column vector of $\mathbf{Z}$, and $\mathbf{o}$ is the zero row vector.

\section{References}

[1] Iakawa, N., Kishinami, T. and Kimura, F. eds. Rapid Product Development, Chapman \& Hall, Proc. 8th Int. Conf. Production Engineering, (1997).

[2] Catmull, E., and Clark, J. Recursively Generated B-Spline Surfaces on Arbitrary Topological Meshes. Computer Aided Design 10, 6 (1978), 350355.

[3] DeRose, T., Kass, M. and Tien T. Subdivision Surface in Character Animation, Computer Graphics Proceedings (SIGGRAPH 98) (1998), 85-94.

[4] Doo, D., and Sabin, M. Analysis of the Behaviour of Recursive Division Surfaces near Extraordinary Points. Computer Aided Design 10, 6 (1978), 356-360.

[5] Dyn, N., Gregory, J. A., and Levin, D. A Four-Point Interpolatory Subdivision Scheme for Curve Design. Comput. Aided Geom. Des. 4 (1987), 257268.

[6] Dyn, N., Levin, D., and Gregory, J. A. A Butterfly Subdivision Scheme for Surface Interpolation with Tension Control. ACM Trans. Gr. 9, 2 (April 1990), 160169.

[7] Halstead, M., Kass, M. and DeRose, T. Efficient, fair interpolation using Catmull-Clark surfaces. Computer Graphics, 27(3):35-44, August 1993.

[8] Hoppe, H., DeRose, T., Duchamp, T., Halstead, M., Jin, H., Mcdonald, J., Schweitzer, J., and Stuetzle, W. Piecewise Smooth Surface Reconstruction. In Computer Graphics Proceedings, Annual Conference Series, (SIGGRAPH '94), 295-302, 1994.

[9] Kobbelt, L. Interpolatory Subdivision on Open Quadrilateral Nets with Arbitrary Topology. In Proceedings of Eurographics 96, Computer Graphics Forum, 409420, 1996.

[10] Kobbelt, L. Interpolatory Subdivision on Open Quadrilateral Nets with Arbitrary Topology, Computer Graphics Forum 15 (1996), Eurographics, '96 issue, pp.409-420. 
[11] Loop, C. Smooth Subdivision Surfaces Based on Triangles. Master's thesis, University of Utah, Department of Mathematics, 1987.

[12] Nasri, A. H. Polyhedral Subdivision Methods for Free-Form Surfaces. ACM Trans. Gr. 6,1 (January 1987), 29-73.

[13] Peters, J., and Reif, U. The Simplest Subdivision Scheme for Smoothing Polyhedra. ACM Trans. Gr. 16(4) (October 1997).

[14] Reif, U. A Unified Approach to Subdivision Algorithms Near Extraordinary Points. Comput. Aided Geom. Des. 12 (1995), 153-174.

[15] Schröder, P. and Zorin, D. et al. Subdivision for Modeling and Animation, ACM Siggraph '98, Course Note No. 36. (1998).

[16] Suzuki, H., Yamamoto, T., Kanai, T. and Kimura, F.: (in Japanese) Triangular Mesh Modelling Based on Welch-Witkin Approximations to Thin Plate Surfaces, J. Japan Society for Precision Engineering, Vol.63, No.1, pp.45-49 (1997).

[17] Yamamoto, T., Suzuki, H., Kanai, T. and Kimura, F.: (in Japanese), Extended Triangular Mesh Subdivision Methods and their Evaluation, The University of Tokyo, Department of Precision Engineering, Kimura \& Suzuki Lab. TR-98-01 (1997).

[18] Welch, W. and Witkin, A. Free-Form Shape Design Using Triangulated Surfaces, Computer Graphics, (1994) 247, (Proc. ACM Siggraph '94).

[19] Zorin, D., Schröder, P., and Sweldens, W. Interpolating Subdivision for Meshes with Arbitrary Topology. Stanford Univ. Computer Science, TR-96-06, (1996)

[20] Zorin, D., Schröder, P., and Sweldens, W. Interpolating Subdivision for Meshes with Arbitrary Topology. Computer Graphics Proceedings (SIGGRAPH 96) (1996), 189-192. 